\title{
The use of dentin autograft in socket presenvation procedure
}

\author{
Elitsa Deliverska ${ }^{1}$, loanna Polichroniadou ${ }^{2}$, \\ Payam Forghani $^{2}$
}

1. Department of Oral and Maxillofacial surgery, Faculty of Dental Medicine, Medical University of Sofia;

2. Students in Faculty of Dental Medicine, Medical University of Sofia;

\begin{abstract}
Regenerative medicine is based on advanced in research in biomaterials one of which is dentin autograft, which can have a major impact on the patient's treatment plan and for improving the quality of life.
\end{abstract}

This review has been designed to investigate the effects of alveolar ridge preservation of alveolar bone with dentin autograft. The success of implant-supported restorations depends on the interaction between a number of anatomical, technical, surgical and prosthetic factors. Restorative-driven implant placement allows the optimal support of the surrounding soft and hard tissues and a satisfactory emergence profile of the final prosthesis. Ridge preservation is any procedure that takes place at the time of, or shortly after an extraction, prior or simultaneously with implant placement to minimise resorption of the ridge and maximise bone formation within the socket.

Alveolar ridge preservation procedures with dentin autograft may decrease the need for further ridge augmentation during implant placement in comparison with $u$

Keywords: dentin autograft, socket preservation, dental implants, ridge preservation nassisted socket healing. 


\section{Background}

Exodontia is indicated when a tooth has a very poor prognosis and cannot be restored/maintained in a satisfactory condition for the patient. Alveolar bone and soft tissue remodelling is a normal physiological response following tooth extraction. These tissue changes have been recorded as leading to a 40-60\% decrease in the height and the width of the residual alveolar ridge, narrowing of the keratinised mucosa and reduction in the volumetric soft tissue thickness The resorption process varies greatly amongst individual patients and tooth position and may be affected by several factors such as the presence of infection, previous periodontal disease, the extent of a traumatic injury and the number or the thickness of the bony socket walls. An equilibrium is reached approximately 3-4 months post-extraction, resulting in a bone and soft tissue level that is lower than that of the neighbouring teeth as complete regeneration of the socket site never occurs. The reduction in horizontal alveolar bone dimensional change was found to be variable. No evidence was identified to clearly indicate the superior impact of a type of ARP intervention (GBR, socket filler and socket seal) on bone dimensional preservation, bone formation, keratinised tissue dimensions and patient complications.(1)

The status of the extraction socket, post exodontia, results in irreversible resorption of the alveolar crest and mainly buccal plate. Extraction sockets without any graft material, result in an average loss of $2.8 \mathrm{~mm}$ of buccal-lingual width and $1.74 \mathrm{~mm}$ in the vertical dimension in the buccal wall height.(2) Thus, it is mandatory that surgical techniques and methods to be executed in order to counteract or at least, to reduce the aforementioned crest resorption. This afunctional atrophy is especially more problematic in patients who are referred for dental implant procedure. Thus the alveolar ridge preservation procedure was created to combat this problem.

Systematic reviews have reported a weighted mean in alveolar ridge reduction in $3.87 \mathrm{~mm}$ in width and $1.67 \mathrm{~mm}$ in height (3) after 3 months of healing or a $63 \%$ and $22 \%$ dimensional loss in a horizontal and vertical plane taking place at the first 6 months after the extraction.(4) The horizontal bone loss/ resorption of the socket is generally more pronounced at the buccal plate, and the vertical resorption is also more evident on the buccal contour of the ridge. $(5,6)$

To reduce the loss of alveolar bone to an acceptable level, several alveolar ridge preservation (ARP) techniques' have been proposed with the purpose to maintenance of the existing soft and hard tissue envelope and stable ridge volume for optimizing functional and esthetic outcomes and also the simplification of treatment procedures subsequent to the ridge preservation.

The preservation and regeneration of the gingival tissue is also important as it helps to establish an ideal functional and aesthetic foundation, before prosthetic rehabilitation occurs.(7-10) Although there is recognition that various ARP techniques can be used to preserve and promote alveolar bone and soft tissue development in the extraction socket area (11-16), heterogeneity of the published data has led, and to conclude that the clinical outcome and prosthetic options available following ARP are inconclusive. 17

The procedure of socket preservation include minimally traumatic extraction of a tooth, followed by immediate grafting of the extraction sockets using particulate bone grafts or substitutes, guided bone regeneration (GBR) with or without bone grafts or substitutes $(18,19,20)$ and a socket seal technique using different tissue graft materials. $(21,22)$ The use of grafting materials as an adjunct to GBR or socket seal techniques is based on the assumption that this material may be useful not only in prohibiting 
membrane or soft tissue graft collapse into the socket area but also in enhancing new bone formation through osteoinduction and osteoconduction.

Direct grafting and augmentation of the extraction socket has been proposed using autogenous bone (23), demineralised freeze-dried bone allograft $(24,25)$, mineralised freeze-dried bone allograft (26), deproteinised bovine bone (27), alloplastic polymers (28), bioactive glasses and composite ceramic materials. Although these bone substitutes were able to maintain the tissue contours in extraction sites, the conservancy of the gingival and bone tissue was variable. Marked differences in the quantity and the quality of the regenerated tissue have been reported, with the presence of the graft sometimes identified as interfering with the normal healing process. $(29,30)$

Some authors develop bioabsorbable dentin materials (31), harmonized with bone remodelling, by using the supersonic and acid-etching technology.

The goal of clinicians in the regenerative field is to search biomaterials that are promoting bone growth which are also eventually being absorbed to be replaced by bone at the same time.(32) Non-absorbable materials and products are never completely replaced by bone and therefore tend to give rise to chronic inflammation as a result of the foreign body and the vitality and 'quality of the new bone is not the same as the normal alveolar bone.(33) Autografts have been proposed as a modern solution to minimize bone resorption after extraction and enhance buccal crest dimensions both vertically and horizontally. Various grafting methods and materials are available, however, none are as straight forward and reliable as the so called golden standard -autograft. Nonconventional autograft procedures have recently gained popularity due to their success, such as the use of autogenous auto-tooth graft materials like dentin. Allografts are also commonly used, albeit, are not as common as autografts due to the more complex nature of the procedure.(34, 35) Post-operative complications after ARP are mainly associated with soft tissue inflammation and infection.

\section{Review results}

Autogenous grafts boast all three perks of biological integration; osteogenic, osteoconductive and osteoinductive properties - whereas the xenografts and aloplastic materials have osteocundution and allografts has osteinduction and osteocuduction. The non-immunogenicity and affordability of autografts heavily outweigh the inconveniences such as, prolonged operation time and risk of donor site morbidity. On the other hand, the mechanical properties of the autografts is superior (36), and can withstand extreme mechanical forces. However, they are more difficult to shape and conform to a bony defect.

Due to the close biochemical similarities between bone and dentin; both contain $10 \%$ fluid, $18 \%$ collagen, $2 \%$ non-collagenous proteins, and $70 \%$ hydroxyapatite in weight volume.(37) Autogenous dentin is divided into the block type and powder type, and is usually obtained from extracted non-functional teeth. Once collected, the dentin is decalcified in $0.6 \mathrm{~N} \mathrm{HCl}$ solution (to form the demineralized dentin matrix), rinsed in cold distilled water and finally freeze dried for storage. These auto-tooth grafts are then immersed in $0.9 \%$ $\mathrm{NaCl}$ solution before use, for 30 minutes, and finally placed in the bony defect. Demineralized dentin matrix (DDM) is an acid insoluble, dentin collagen that is absorbable but difficult to digest by the organism. Advantages of DDM is the natural ability to coagulate blood plasmas, and the high success rate of the procedure. Additionally the procedure of PRF and PRP could be done. Blood from each patient is 
centrifuged and the middle layer is used as fibrin glue (so called concentrated growth factors - CGF). A composite of DDM granules and CGF glue stimulate the regeneration of the bone and can lead to a better prognosis for dental implants. $(38,39,40)$

Allografts are also a promising variant for patients but has some disadvantages. They have proven to be brittle, poorly processable into porous form, and are unable to generate structures tailored to the specific needs of the patient. Allogenic grafts has osteoinduction properties and above the layer of allograft the clinician shoul put layer of xenograft material to keep the space for longer. The risk of transfer of diseases is very low.

\section{Conclusion}

In the paradigm of alveolar ridge preservation, conventional methods such as autografts and allografts with non-synthetic material have been the most popular and successful techniques. With the discovery of autogenous auto-tooth grafts, clinicians and patients can recycle non-functional or extracted teeth to fill bony defects and augment alveolar bone with very good prognosis.

\section{References}

1. MacBeth N, Trullenque-Eriksson A, Donos $\mathrm{N}$ et al., Hard and soft tissue changes following alveolar ridge preservation: a systematic review. Clin. Oral Impl. Res. 00, 2016, 1-23.

2. Parker $\mathrm{C}$. The growth of implant dentistry. The dentist. Surrey (United Kingdom): George Warman Publications; 2012.

3. Van der Weijden F, Dell'Acqua F, Slot DE, Alveolar bone dimensional changes of post-extraction sockets in humans: a systematic review. J Clin Periodontol 2009; 36: 1048-1058.

4. Pagni, G., Pellegrini, G., Giannobile, W. V. et al., Postextraction Alveolar Ridge Preservation: Biological Basis and Treatments. International Journal of Dentistry, 2012, 1-13.

5. Pietrokovski, J. and Massler, M. Alveolar Ridge Resorption Following Tooth Extraction. Journal of Prosthetic Dentistry, 1967, 17, 21-27.

6. Araujo, M. G., Lindhe, J. Dimensional ridge alterations following tooth extraction. An experimental study in the dog. Journal of Clinical Periodontology, 2005, 32(2), 212-218.

7. Seibert, J.S., Reconstruction of deformed, partially edentulous ridges, using full thickness onlay grafts. Part II. Prosthetic/periodontal interrelationships.1983, 4(6):549-562.

8. Struder, L. Enhanced proliferation, survival, and dopaminergic differentiation of CNS precursors in lowered oxygen, 2000, 1;20(19):7377-7383.

9. Jung, R. E, Postextraction tissue management: a soft tissue punch technique, 2004, 24(6):545553.

10. Prato, GP., Prevention of alveolar ridge deformities and reconstruction of lost anatomy: a review of surgical approaches, 2004, 24(5):434-445.

11. Vignoletti, F., Matesanz, P., Rodrigo, D. et al., Surgical protocols for ridge preservation after tooth extraction. A systematic review. Clinical Oral Implants Research, 2011, 23, 22-38.

12. Wang, R. E., Lang, N. P., Ridge preservation after tooth extraction. Clinical Oral Implants Research, 2012, 23, 147-156.

13. De Risi, V., Clementini, M., Vittorini, G. et al., Alveolar ridge preservation techniques: a systematic review and meta-analysis of histological and histomorphometrical data. Clinical Oral Implants Research, 2013, 26(1), 50-68. 
14. Horváth, A., Mardas, N., Mezzomo, L. A. et al., Alveolar ridge preservation. A systematic review. Clinical Oral Investigations, 2012, 17(2), 341-363.

15. Avila-Ortiz, G., Elangovan, S., Kramer, K. W. O. et al., Effect of Alveolar Ridge Preservation after Tooth Extraction. Journal of Dental Research, 2014, 93(10), 950-958.

16. Morjaria, K. R., Wilson, R., Palmer, R. M., Bone Healing after Tooth Extraction with or without an Intervention: A Systematic Review of Randomized Controlled Trials. Clinical Implant Dentistry and Related Research, 2012, 16(1), 1-20.

17. Mardas, N., Trullenque-Eriksson, A., MacBeth, N. et al., Does ridge preservation following tooth extraction improve implant treatment outcomes: a systematic review. Clinical Oral Implants Research, 2015, 26, 180-201.

18. Adriaens, P.A., Van der Stede, N, Alveolar bone protection of extraction sites with a resorbable bilayer GBR membrane. Journal of Dental Research, 1998, 77: 777-790.

19. Iasella, J. M., Greenwell, H., Miller, R. L. et al., Ridge Preservation with Freeze-Dried Bone Allograft and a Collagen Membrane Compared to Extraction Alone for Implant Site Development: A Clinical and Histologic Study in Humans. Journal of Periodontology, 2003, 74(7), 990-999.

20. Mardas, N., Chadha, V., Donos, N., Alveolar ridge preservation with guided bone regeneration and a synthetic bone substitute or a bovine-derived xenograft: a randomized, controlled clinical trial. Clinical Oral Implants Research, 2010, 21(7), 688-698.

21. Lekovic, V., Camargo, P. M., Klokkevold, P. R. et al., Preservation of Alveolar Bone in Extraction Sockets Using Bioabsorbable Membranes. Journal of Periodontology, 1998, 69(9), 1044-1049.

22. Bartee, B. K. Extraction Site Reconstruction for Alveolar Ridge Preservation. Part 1: Rationale and Materials Selection. Journal of Oral Implantology, 2001, 27(4), 187-193.

23. Lundh, L.-G., Czyzykow, S., Öst, L.-G. Explicit and implicit memory bias in panic disorder with agoraphobia. Behaviour Research and Therapy, 1997, 35(11), 1003-1014.

24. Becker $\mathrm{J}$ et al., Functional interaction of cytosolic hsp70 and a DnaJ-related protein, Ydj1p, in protein translocation in vivo. Mol Cell Biol, 1996,16(8):4378-4386.

25. Froum, S., Cho, S.-C., Rosenberg, E. et al., Histological Comparison of Healing Extraction Sockets Implanted With Bioactive Glass or Demineralized Freeze-Dried Bone Allograft: A Pilot Study. Journal of Periodontology, 2002, 73(1), 94-102.

26. Feuille. F., Clinical and histologic evaluation of bone-replacement grafts in the treatment of localized alveolar ridge defects. Part 2: bioactive glass particulate. 2003, 23(2):129-137.

27. Artzi, Z., Tal, H., Dayan, D. Porous Bovine Bone Mineral in Healing of Human Extraction Sockets. Part 1: Histomorphometric Evaluations at 9 Months. Journal of Periodontology, 2000, 71(6), 1015-1023.

28. Serino, G. Ridge preservation following tooth extraction using a polylactide and polyglycolide sponge as space filler: a clinical and histological study in humans.2003, 14(5):651-658.

29. Horvath, S. DNA methylation age of human tissues and cell types. Genome Biology, 2013, 14(10), R115.

30. Monje A., Pikos M. A., Chan et al., On the Feasibility of Utilizing Allogeneic Bone Blocks for Atrophic Maxillary Augmentation. BioMed Research International, 2014, 1-12.

31. Murata M., Sato D., Hino J. et al, Acid-insoluble human dentin as carrier material for recombinant human BMP-2. Journal of Biomedical Materials Research Part A, 2011, 100A(3), 571-577.

32. Rabelo GD, de Paula PM, Rocha FS et al., Retrospective study of bone grafting procedures before implant placement. Implant Dent, 2010;19(4):342-350.

33. Evian $\mathrm{Cl}$, Rosenberg ES, Coslet JG et al, The osteogenic activity of bone removed from healing extraction sockets in humans J. Periodontol, 1982;53:81-85.

34. Barone A, Ricci M, Tonelli P et al., Tissue changes of extraction sockets in humans: a comparison of spontaneous healing vs ridge preservation with secondary soft tissue healing. Clin Oral Impl Res 2013;24:1231-1237.

35. Moghaddas H, Amjadi MR, Naghsh N. Clinical and biometrical evaluation of socket preservation using demineralized freeze-dried bone allograft with and without the palatal connective tissue as a biologic membrane.Dent Res J 2012;9:758-763. 
36. Thalmair T, Fickl S, Schneider D, Hinze M, Wachtel H. Dimensional alterations of extraction sites after different alveolar ridge preservation techniques-a volumetric study. J. Clin Periodontol, 2013;40:721-727.

37. Bang. G. \& Urist, MR. Bone inductive in excavation chambers in matrix of decalcified dentin. Arch Surg, 1967, 94 (6), 781-789.

38. Akazawa,T., Murata, M., Sasaki, T. et al., Biodegradation and bioabsorption innovation of the functionally graded cattle-bone-originated apatite with blood compatibility. J Biomed Mater Res, 2006, 76A, 1, 44-51.

39. Murata, M. Autogenous demineralized dentin matrix for maxillary sinus augmentation in human. The first clinical report. 81th International Association for Dental Research, Geteburg, Sweden, 2003, June.

40. Murata, M., Akazawa, T., Mitsugi, M. et al., Autograft of Dentin Materials for Bone Regeneration. Advances in Biomaterials Science and Biomedical Applications, 2013, 15, 391-403.

\section{Corresponding author:}

Associate prof. Elitsa Deliverska, PhD

Faculty of Dental medicine,

Medical University Sofia

Department of Oral and Maxillofacial surgery

Sofia 1431 Str."Georgi Sofiiski"1 